\title{
NY-ESO-1 expression in hepatocellular carcinoma: A potential new marker for early recurrence after surgery
}

\author{
HENG XU ${ }^{1,2^{*}}, \mathrm{NA} \mathrm{GU}^{1 *}$, ZHAO-BO LIU ${ }^{1}$, MIN ZHENG $^{3}$, FANG XIONG $^{1}$, \\ SI-YING WANG ${ }^{2}$, NING LI ${ }^{1}$ and JUN LU ${ }^{1}$ \\ ${ }^{1}$ Tumor Biotherapy Ward of Beijing YouAn Hospital, Capital Medical University, Beijing 100069; \\ ${ }^{2}$ Department of Pathophysiology, Anhui Medical University, Hefei 230032; \\ ${ }^{3}$ Hangzhou High Throughput Drug Screening Center, SanDun, Hangzhou 310030, P.R. China
}

Received April 20, 2011; Accepted August 5, 2011

DOI: $10.3892 / \mathrm{ol} .2011 .441$

\begin{abstract}
NY-ESO-1 belongs to the cancer testis antigens (CTA) family, and is identified in a variety of tumors. Certain studies have demonstrated that NY-ESO-1 predicts tumor recurrence and treatment response. No reports are currently available regarding the correlation between NY-ESO-1 and the recurrence of hepatocellular carcinoma (HCC) following surgery. The purpose of the present study was to evaluate the association between NY-ESO-1 and relapse of HCC and to explore the possible mechanisms for this correlation. A total of $120 \mathrm{HCC}$ patients were analyzed for the expression of NY-ESO-1 by immunohistochemistry (IHC). A stable NY-ESO-1 over-expressed HepG2 cell line (ESO-HepG2) was established to determine the biological effects of NY-ESO-1 on cell proliferation, cell cycle and migration by using the xCELLigence DP system, flow cytometry and xCELLigence SP system. NY-ESO-1 was positive in 28 of 120 (23.3\%) HCC tumor tissues. NY-ESO-1 was not detectable in adjacent normal liver tissues. A close correlation was found between NY-ESO-1 expression and the recurrence of HCC following surgery $(\mathrm{P}=0.007)$. Kaplan-Meier analysis showed a shorter recurrence-free survival (RFS) for patients positive for NY-ESO-1 (log-rank test, $\mathrm{P}=0.003$ ). The Cox regression model demonstrated that NY-ESO-1 expression was a significant independent predictor for the recurrence of $\mathrm{HCC}$ following curative surgery $(\mathrm{P}=0.022)$. Compared with HepG2 cells, ESO-HepG2 cells have increased migration but not proliferation ability. In conclusion, NY-ESO-1 expression is associated with worse HCC outcome following surgery, and the mechanism for this finding may be that NY-ESO-1 increases tumor cell migration.
\end{abstract}

Correspondence to: Dr Jun Lu, Tumor Biotherapy Ward of Beijing YouAn Hospital, Capital Medical University, Beijing 100069, P.R. China

E-mail: lujun98@gmail.com

*Contributed equally

Key words: NY-ESO-1, hepatocellular carcinoma, early recurrence

\section{Introduction}

Hepatocellular carcinoma (HCC) is the fifth most common cancer in the world and one of the most prevalent malignancies in Asia (1). Current curative treatment options include surgical resection and liver transplantation. Liver transplantation is effective only in the early stages of disease. Surgery is possible in few patients and curative in only a small percentage, due to recurrence following the surgery (2). Besides exploring effective therapeutic methods, investigators are attempting to find biomarkers to predict tumor recurrence. The NY-ESO-1 antigen was originally found in esophageal cancer by serological recombinant cDNA expression cloning (SEREX) and belongs to the cancer/testis antigen (CTA) family (3). NY-ESO-1 expression is restricted to testicular germ cells in normal adult tissues and is found in numerous malignancies including malignant melanoma, hepatoma, breast and lung cancer. The NY-ESO-1 protein is known to be markedly immunogenic for numerous advanced and metastatic types of cancer $(4,5)$. NY-ESO-1 is a potential biomarker for the prediction of tumor recurrence and treatment outcomes in a variety of tumors, including gastrointestinal stromal tumors (6) and cutaneous melanoma (7).

Although a number of studies showed inconsistently that NY-ESO-1 is positive in certain HCCs (8-16), two studies have demonstrated that NY-ESO-1 correlates with the metastasis of HCC $(11,12)$. However, the role of NY-ESO-1 in the prognosis of HCC following surgery remains unclear. The present study used immunohistochemistry (IHC) to evaluate the NY-ESO-1 expression in tissues from 120 HCC patients, the correlation between the patients' clinical parameters and recurrence-free survival (RFS). In addition, an NY-ESO-1 overexpressing cell line was used to explore the effect of NY-ESO-1 on cell behavior.

\section{Materials and methods}

Patients and specimens. Between August 2004 and March 2008, 120 consecutive patients who underwent curative resection or liver transplantation with no microscopic evidence of residual tumor were included in this study. All 120 patients had histologically proven HCC. The inclusion criteria for the 
study were: i) the absence of extrahepatic metastasis; ii) curative resection defined as histological evidence of the complete removal of HCC tumors; and iii) no additional therapies or multi-modality treatment for HCC until the development of recurrence. Patients diagnosed with a mixed tumor containing HCC and cholangiocarcinoma components were excluded from the study.

Follow-up. Patients were regularly followed up at the outpatient clinic (every 1-3 months). The endpoint was recurrence or November 30, 2009. The clinical profiles were age, gender, HBsAg, Child-Pugh classification, surgery (liver transplantation and curative resection), primary HCC lesion [timing of diagnosis, size, number, gross major vessel invasion (portal vein), tumor distribution and histological differentiation], and tumor relapse (time). The study conformed to the tenets of the Declaration of Helsinki and informed consent was obtained from all patients prior to the study.

Assessment. Patients were monitored by serum $\alpha$-fetoprotein (AFP), abdomen ultrasound and chest X-ray every 1 to 6 months, according to the postoperative time. Tumor recurrences were confirmed by computed tomography (CT), and, if necessary, magnetic resonance imaging (MRI). RFS was calculated from the date of surgery until the date of recurrence.

Immunohistochemistry (IHC). Tumor samples were retrieved from the archives of the Department of Pathology at YouAn Hospital, Beijing, China. Serial formalin-fixed, paraffin-embedded tumor samples were deparaffinized in xylene and rehydrated in a series of graded alcohols and distilled water. Endogenous peroxidase activity was quenched by $3 \% \mathrm{H}_{2} \mathrm{O}_{2}$. Prior to immunostaining, sections were incubated for $12 \mathrm{~min}$ in citrate buffer $(\mathrm{pH}$ 6.0) in a microwave oven at $99^{\circ} \mathrm{C}$ to enhance the immunoreactivity. Following blocking, the sections were incubated at $4^{\circ} \mathrm{C}$ overnight with mouse monoclonal NY-ESO-1 antibody (E978 clone; Invitrogen, Carlsbad, CA, USA). Detection was carried out using the streptavidin-biotin-peroxidase method with a PV-9000 kit according to the manufacturer's instructions. The DAB system was employed as a chromogen. Normal adult testis with intact spermatogenesis served as positive controls. Slides were counterstained with hematoxylin and mounted. Normal liver tissues served as negative controls. Immunoreactivity of tumor cells was graded based on the amount of immunopositive tumor cells as follows:,$+++>67 \%$ of cells stained;,$++ 33-67 \%$; + $5-33 \%$; focal $<5 \%$ and - , no staining cells. Focal staining was also considered as negative.

Cell line and construction of NY-ESO-1-expressing plasmid. To determine the role of NY-ESO-1 in HCC, we established stably transfected NY-ESO-1-overexpressing HepG2 cells (ESO-HepG2) and evaluated their proliferation and migration behaviors. Briefly, HepG2 (HCC) cells were obtained from the American Type Culture Collection and cultured in DMEM supplemented with antibiotics/antimycotics and 10\% fetal bovine serum (FBS). The NY-ESO-1 cDNA encoding NY-ESO-1 was amplified by PCR from mRNA and subcloned into a pcDNA3.1 expression vector (Invitrogen) to form plasmid-designated pcDNA3.1-ESO.
Transfection and selection of stably transfected ESO-HepG2 cells. Subconfluent HepG2 cells (70-80\%) were transfected with $2 \mu \mathrm{g}$ of pcDNA3.1-ESO and pcDNA3.1 using Lipofectamine 2000 (Invitrogen) according to the manufacturer's instructions. For stable transfection, the cells were exposed to $400 \mu \mathrm{g} / \mathrm{ml} \mathrm{G} 418$ (Invitrogen) after 1 day of transfection. Four weeks later, the cells were plated at a lower density in DMEM with $250 \mu \mathrm{g} / \mathrm{ml} \mathrm{G} 418$ and $10 \%$ FBS in 96-well plates until a single colony was formed. Single cloned cells designated as ESO-HepG2 cells were isolated and grown. RT-PCR was used to test NY-ESO-1 expression.

Cell cycle and proliferation analysis. Cells were diluted and seeded at $6 \times 10^{5} / \mathrm{ml}, 1 \times 10^{5} / \mathrm{ml}$ and $2.5 \times 10^{4} / \mathrm{ml}$ in ACEA's x96 microtiter plates in $100 \mu \mathrm{l}$ of culture medium. Cell proliferation was continuously monitored every $15 \mathrm{~min}$ using the XCELLigence for a period of 24-72 h via calculation of a 'cell index' (to reflect the surface area covered by the cells) for each plate (Roche, Indianapolis, IN, USA) (17). Following synchronization, cells were collected, fixed and stained with propidium iodide. The cell cycle distribution was determined using a BD FACSCalibur flow cytometer and Cell Quest software.

Cell migration assay. Cells were seeded at $6 \times 10^{5} / \mathrm{ml}$ in $100 \mu 1$ of serum-free medium in the upper chamber with an $8 \mu \mathrm{m}$ pore size, and the lower chamber contained $10 \%$ serum. Cell migration was continuously monitored every 15 min using the xCELLigence for a period of $24 \mathrm{~h}$ via calculation of a 'cell index' (to reflect the surface area covered by the cells) for each plate (Roche).

Statistical analysis. The $\chi^{2}$ test (including Fisher's exact test) and independent sample t-test were employed for comparison between groups. A RFS curve was plotted using the Kaplan-Meier method. A statistical comparison of the RFS was performed using the log-rank test. A multivariate analysis using the Cox proportional hazard model was used to identify the independent risk factors for tumor recurrence. $\mathrm{P}<0.05$ was considered to be statistically significant. SPSS 11.5 was used for all analyses.

\section{Results}

Baseline characteristics of the patients. Of the $120 \mathrm{HCC}$ patients, 79 were successfully followed up, 21 refused to cooperate and 20 were lost to follow-up. The median follow-up period was 19 months (range 1.0-61.0). Given the possibility of selection bias, we compared the baseline characteristics of the 79 and 41 cases, as shown in Table I. No significant difference was found in any of the indices, such as age, gender, HBV infection, serum AFP, Child-Pugh Score and the pathologic parameters including tumor size and number, portal vein thrombosis and histological differentiation. Thus, the data from the 79 patients represent the entire cohort. Of the 79 patients, 11 succumbed to other diseases. As there was no evidence of tumor relapse, these patients were also included in the analysis.

NY-ESO-1 expression in HCCs. NY-ESO-1 staining was positive in the testis epithelial cells (Fig. 1A), but not in normal liver tissue (Fig. 1B). A total of 28 out of 120 HCC specimens were positive $(23.3 \%)$. Among them, one was graded as 
Table I. The baseline characteristics of 79 cases with follow-up and 41 cases without follow-up.

\begin{tabular}{lcccr}
\hline & \multicolumn{3}{c}{120 cases } & \\
\cline { 2 - 4 } Clinical parameters & 79 cases (\%) & 41 cases (\%) & $\chi^{2}$ & P-value \\
\hline NY-ESO-1 expression (positive) & 19.0 & 31.7 & 2.441 & 0.118 \\
Gender (male) & 82.3 & 78.0 & 0.312 & 0.557 \\
Age $<52$ years & 45.6 & 41.5 & 0.185 & 0.667 \\
Tumor size $\geq 5$ cm & 51.9 & 56.1 & 0.191 & 0.662 \\
Tumor number (120) multinodular & 40.5 & 34.1 & 0.462 & 0.497 \\
Portal vein thrombosis (with) & 24.4 & 36.6 & 1.968 & 0.161 \\
Abnormal serum AFP & 78.1 & 84.2 & 0.591 & 0.442 \\
Transplantation surgery & 58.2 & 56.1 & 0.137 & 0.823 \\
HBsAg-positive & 75.0 & 71.8 & 0.072 & 0.711 \\
Child-Pugh Score & & & & 0.964 \\
A & 61.4 & 60.7 & & 0.370 \\
B & 12.3 & 14.3 & & \\
C & 26.3 & 25.0 & & \\
Histological differentiation & & & & \\
Well & 52.8 & 53.8 & & \\
Moderately & 30.6 & 38.5 & & \\
Low & 16.6 & & & \\
\hline
\end{tabular}

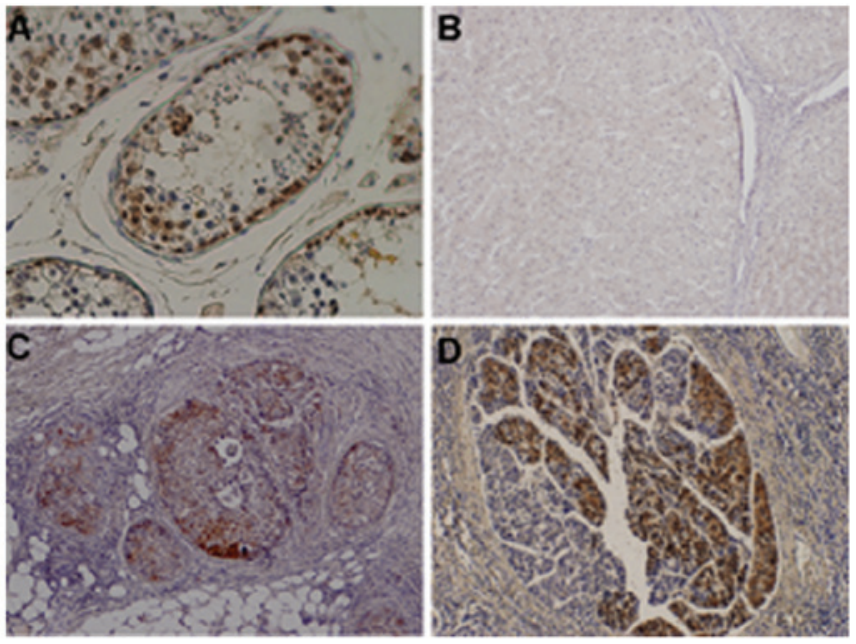

Figure 1. Immunohistological staining with monoclonal antibody E978 to NY-ESO-1. (A) NY-ESO-1 antigen expression in the testis epithelial cells (10x20) as a positive control and (B) normal liver tissue (10x10) as a negative control. (C) Cytoplasmic staining of tumor cells from one patient (10x10) and (D) heterogeneous cytoplasmic and nuclear staining of tumor cells from one patient $(10 \times 10)$.

,+++ 7 as ++ and 20 as + (Fig. 1C and D). NY-ESO-1 was located predominantly in the cytoplasm (Fig. 1C), although the nucleus was also stained in a few cases (Fig. 1D). Notably, even in the same specimen, certain areas were positive, whereas other areas were negative. No staining was observed in the tissue adjacent to HCC.

Correlation between NY-ESO-1 expression and recurrence in HCC. In 13 out of 38 tumor recurrence patients NY-ESO-1 was found to be positive (34.2\%, Table II), whereas in 2 of 30 non-recurrence cases NY-ESO-1 was positive $(6.7 \%)$. The difference was statistically significant $(\mathrm{P}=0.007)$. Of the 68 patients who completed follow-up, the median RFS was 19 months. Regarding NY-ESO-1-positive patients, it was found that in $73.3 \%$ of them the RFS was $<19$ months, and in $26.7 \%$ it was $\geq 19$ months. On the other hand, in the NY-ESO1-negative patients, $41.5 \%$ had an RFS $<19$ months, while in $58.5 \%$ it was $\geq 19$ months $(\mathrm{p}=0.027)$

Using the Kaplan-Meier method, we observed that the median RFS was 12 months in NY-ESO-1-positive patients, whereas in NY-ESO-1-negative patients the RFS was estimated to be 40-50 months (the endpoint blocked the continuation of the follow-up, log-rank test, $\mathrm{P}=0.003$, Fig. 2).

Various clinicopathological parameters were also evaluated for their association with HCC recurrence (Table II). A total of $63.2 \%$ patients who were NY-ESO-1-positive relapsed following liver transplantation compared with $36.8 \%$ of those who were NY-ESO-1-negative $\left(\chi^{2}\right.$ test, $\left.\mathrm{P}=0.033\right)$. The total recurrence rate in NY-ESO-1-positive patients was $86.2 \%$, while this rate was $38.1 \%$ in NY-ESO-1-negative cases $(\mathrm{P}=0.002)$. Additionally, $36.4 \%$ of NY-ESO-1-positive cases were well-differentiated, whereas in NY-ESO-1-negative cases this rate was $65.6 \%\left(\chi^{2}\right.$ test, $\left.\mathrm{P}=0.040\right)$. Other parameters, such as age, gender, HBV infection, serum AFP, tumor size and number and portal vein thrombosis had no role in prognosis.

Multivariate analysis demonstrated that the type of surgery $(\mathrm{P}<0.001)$ and NY-ESO-1 expression $(\mathrm{P}=0.022)$ were independent predictors for the recurrence of $\mathrm{HCC}$ following curative surgery (Table III). When using the forward method and backward method, we found the same results. 
Table II. Univariate analysis of factors affecting recurrence of HCC.

\begin{tabular}{|c|c|c|c|c|}
\hline \multirow[b]{2}{*}{ Clinical parameters } & \multicolumn{2}{|c|}{ Recurrence (68 cases) } & \multirow[b]{2}{*}{$\chi^{2}$} & \multirow[b]{2}{*}{ P-value } \\
\hline & $\begin{array}{c}30 \text { cases } \\
\text { No }(\%)\end{array}$ & $\begin{array}{l}38 \text { cases } \\
\text { Yes }(\%)\end{array}$ & & \\
\hline NY-ESO-1 expression (positive) & 6.7 & 34.2 & 7.398 & 0.007 \\
\hline Gender (male) & 73.3 & 86.8 & 1.979 & 0.160 \\
\hline Age $<52$ years & 60.0 & 52.6 & 0.369 & 0.543 \\
\hline Tumor size $\geq 5 \mathrm{~cm}$ & 53.3 & 55.3 & 0.025 & 0.874 \\
\hline Tumor number (120) multinodular & 36.7 & 36.8 & 0.000 & 0.988 \\
\hline Portal vein thrombosis (with) & 24.1 & 21.1 & 0.090 & 0.764 \\
\hline Abnormal serum AFP & 73.1 & 83.8 & 1.069 & 0.301 \\
\hline Transplantation surgery & 36.8 & 63.2 & 10.719 & 0.001 \\
\hline HBsAg-positive & 72.4 & 80.6 & 0.600 & 0.439 \\
\hline Child-Pugh Score & & & 12.600 & 0.002 \\
\hline $\mathrm{A}$ & 38.1 & 86.2 & & \\
\hline B & 19.0 & 3.4 & & \\
\hline $\mathrm{C}$ & 42.9 & 10.4 & & \\
\hline Histological differentiation & & & 6.440 & 0.040 \\
\hline Well & 65.6 & 36.4 & & \\
\hline Moderately & 17.2 & 45.5 & & \\
\hline Low & 17.2 & 18.2 & & \\
\hline
\end{tabular}

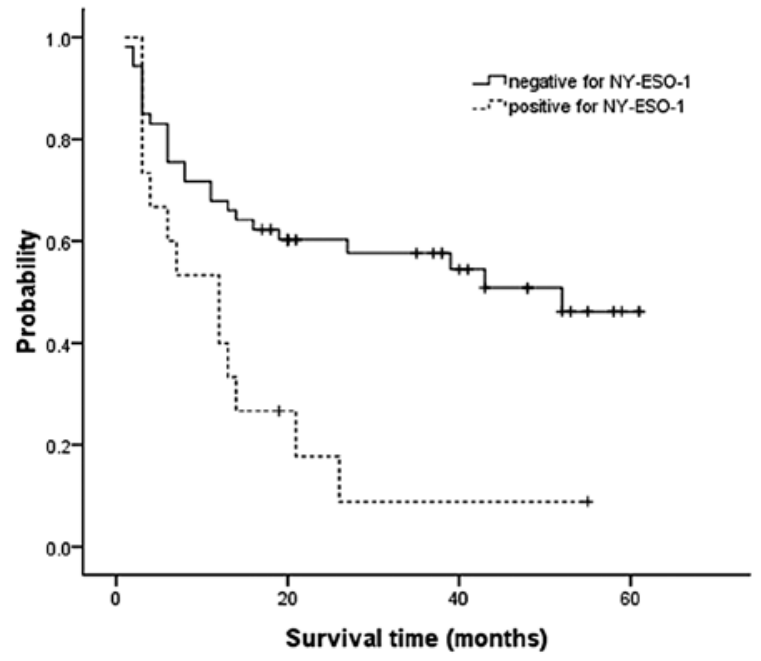

Figure 2. Recurrence-free survival (RFS) according to the NY-ESO-1 immunohistochemistry reaction (Kaplan-Meier method). RFS in HCC patients negative for NY-ESO-1 expression $(n=53)$ and in HCC patients positive for NY-ESO-1 expression $(n=15)$. Log-rank test, $\mathrm{P}=0.003$.

NY-ESO-1 modulates the migration but not proliferation of HepG2 cells. The cell growth curves of HepG2 cells were comparable to those of ESO-HepG2 cells even when cells were seeded to the E-plate with three different densities (Fig. 3A-C). Flow cytometry showed that the cell cycle distributions of these cells were similar (Fig. 3D). ESO-HepG2 cells migrated more compared with HepG2 cells $(\mathrm{P}=0.002$, Fig. 3E).

\section{Discussion}

To the best of our knowledge, the present study is the first to demonstrate that NY-ESO-1 is an independent prognostic factor in $\mathrm{HCC}$, and that the mechanism may involve the enhancement of cancer cell migration.

NY-ESO-1 belongs to the CTA family. It was found in a variety of malignant tumors, such as melanoma, prostate, breast and lung cancer. Furthermore, NY-ESO-1 is demonstrated to be a prognostic marker in certain tumors, including malignant melanoma and gastrointestinal tumors. However, the correlation between NY-ESO-1 and HCC remains unclear. Previous studies showed that the NY-ESO-1-positive rate is from $0-43.9 \%$ in HCC patients (8-16). Certain studies demonstrated that NY-ESO-1 enhanced metastasis in HCC. However, the mechanism remains unclear. There is no detailed study on the predictive value of NY-ESO-1 on HCC recurrence. The present study found that the NY-ESO-1positive rate in this cohort is $23.3 \%$. NY-ESO-1 is a promising antigen for HCC-specific immunotherapy given that it is the most immunogenic CT antigen identified thus far and induces tumor-specific humoral and cellular immune responses to NY-ESO-1 in HCC (10). Although international clinical trials of NY-ESO-1 protein vaccine therapy for multiple types of cancer including malignant melanoma, non small cell lung, ovarian, breast and bladder cancer were conduted, the potential immunotherapy in HCC remains to be evaluated. Understanding the NY-ESO-1 protein expression in HCC is the first step. The finding that NY-ESO-1 is expressed with relatively high frequency in $\mathrm{HCC}$ patients may be of clinical significance. 
Table III. Multivariate analysis of factors affecting recurrence of HCC.

\begin{tabular}{lccr}
\hline Clinical parameters & B & HR (95\% CI) & P-value \\
\hline NY-ESO-1 (positive vs. negative) & 0.980 & $2.664(1.150-6.169)$ & 0.022 \\
Surgery (resection vs. transplantation) & 2.256 & $9.549(3.214-28.370)$ & $<0.001$ \\
Age & -0.052 & $0.938(0.898-0.980)$ & 0.004 \\
\hline
\end{tabular}

HR, hazard ratio; CI, confidence interval.

A

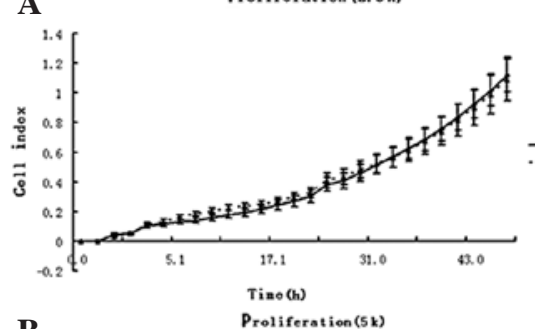

B

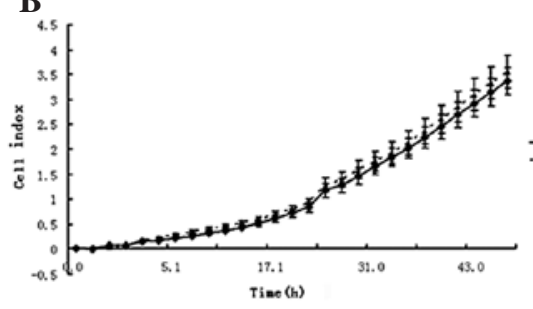

C

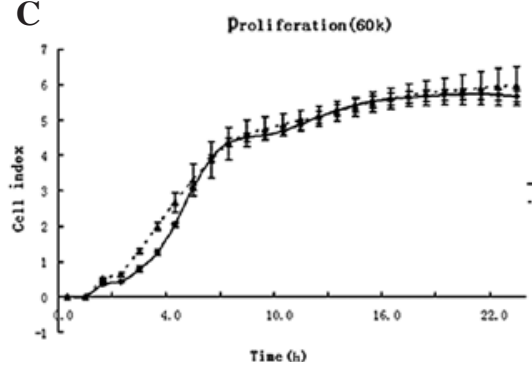

D

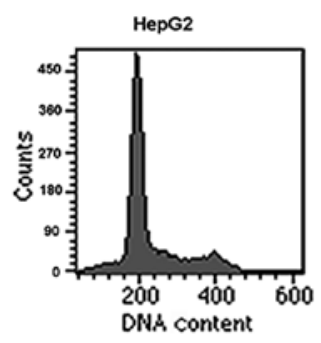

ESO-HepG2

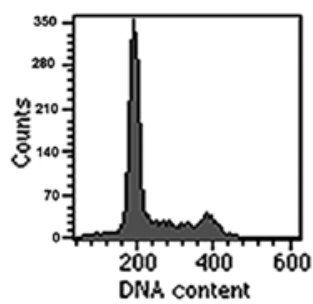

\begin{tabular}{|l|l|l|l|l|l|}
\hline Name & Dip G0-G1 & Dip G2-M & Dip S & G2/G1 & Apoptosis \\
\hline HepG2 & $67.47 \%$ & $4.31 \%$ & $28.21 \%$ & 2.05 & $3.96 \%$ \\
\hline ESO-HepG2 & $66.55 \%$ & $8.51 \%$ & $24.94 \%$ & 1.99 & $1.70 \%$ \\
\hline
\end{tabular}

$\mathbf{E}$

Migration $(60 \mathrm{k})$

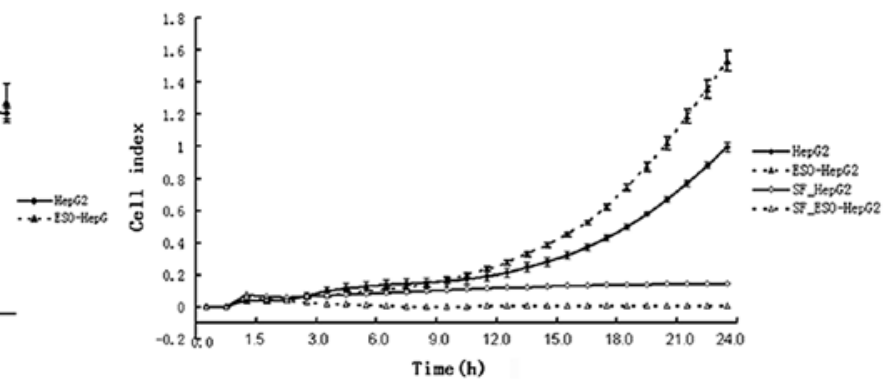

Figure 3. Proliferation curve, cell cycle distribution and migration curve of HepG2 and ESO-HepG2 cells. (A) Dynamic monitoring (Voltage $2.5 \mathrm{~K}$ ) of in vitro cell proliferation using the xCELLigence SP system showed a similar proliferation rate of ESO-HepG2 (HepG2 cells over-expressed with NY-ESO-1) and control HepG2 cells (three different cell densities were seeded to the E-plate); (B) Dynamic monitoring (Voltage $10 \mathrm{~K}$ ); (C) Dynamic monitoring (Voltage 60K); and (D) DNA content of HepG2 and ESO-HepG2 cell lines using flow cytometry. (E) Dynamic monitoring (Voltage $60 \mathrm{~K}$ ) of in vitro cell migration using the xCELLigence DP system showed a higher migration rate of ESO-HepG2 compared with control HepG2 cells. SF_ HepG2, serum-free HepG2; SF_ESO-HepG2, serum-free ESO-HepG2.

Besides the positive rates, we have two new findings: one is that NY-ESO-1 is a prognostic marker in HCC. NY-ESO1-positive patients are 7.28 (odds ratio) times more likely to relapse compared with NY-ESO-1-negative cases following surgery. It appears that the higher the NY-ESO-1 expression, the more likely the recurrence. Another finding is that NY-ESO-1-positive patients have a shorter RFS than that of NY-ESO-1-negative patients. Our data are consistent with those of Perez et al in gastrointestinal stromal tumors (18). Univariate and multivariate analysis clearly indicated that patients with NY-ESO-1-positive tumors have a shorter RFS, which is in accordance with the results of the majority of the previous studies showing that NY-ESO-1 expression may be a biomarker of poor prognosis in numerous types of cancer. For example, the expression of NY-ESO-1 protein has been reported to be correlated with the metastasis of HCC (12). In non-small cell lung cancer, NY-ESO-1 expression significantly increased with the advancement of disease stage in the TNM classification, particularly that related to lymph node metastasis (19). In addition, NY-ESO-1 is more frequently expressed in metastatic than in primary malignant melanoma and its expression is associated with advanced stage (20). The point to be considered is the manner in which NY-ESO-1 increases tumor recurrence and reduces RFS. Two ways in which this can be achieved are possible: one is that NY-ESO-1 enhances tumor cell migration and, therefore, metastasis; another is that NY-ESO-1 stimulates tumor cell growth. Zhou et al found that the larger the HCC, the higher the NY-ESO-1 expression (21). Other authors found no correlation between NY-ESO-1 expression and HCC size $(11,12)$. To clarify the mechanism 
of NY-ESO-1 and HCC behavior, we transfected HepG2 cells with pcDNA3.1-ESO. We found that over-expression of NY-ESO-1 enhanced cell migration, but not proliferation.

In conclusion, the present study confirmed NY-ESO-1 expression in a certain number of HCC patients, and showed that NY-ESO-1 is a promising target for immunotherapy based on its relatively high frequency expression in HCC. NY-ESO-1 may be a potential biomarker for early recurrence and, therefore, a shorter RFS following surgery. Moreover, NY-ESO-1 enhanced tumor cell migration and, therefore, metastasis.

\section{Acknowledgements}

We thank Dr Jan J. Melenhorst at the National Institute of Health for reading and editing the manuscript. We thank our colleague XinQiu Yu (Branch of Liver Surgery, Beijing YouAn Hospital) for help during HCC case collection. This study was supported by the Beijing Natural Science Foundation (7092044-J.L), the National High Technology Research and Development Program of China (No. 2007AA02Z151) and (2009zx10004-309).

\section{References}

1. Parkin DM, Bray F, Ferlay $\mathrm{J}$ and Pisani P: Global cancer statistics, 2002. CA Cancer J Clin 55: 74-108, 2005.

2. Venook AP: Treatment of hepatocellular carcinoma: too many options? J Clin Oncol 12: 1323-1334, 1994.

3. Chen YT, Scanlan MJ, Sahin U, et al: A testicular antigen aberrantly expressed in human cancers detected by autologous antibody screening. Proc Natl Acad Sci USA 94: 1914-1918, 1997.

4. Chen YT, Gure AO, Tsang S, et al: Identification of multiple cancer/testis antigens by allogeneic antibody screening of a melanoma cell line library. Proc Natl Acad Sci USA 95: 6919-6923, 1998.

5. Wang RF,Johnston SL, Zeng G, Topalian SL,Schwartzentruber DJ and Rosenberg SA: A breast and melanoma-shared tumor antigen: T cell responses to antigenic peptides translated from different open reading frames. J Immunol 161: 3598-3606, 1998.

6. Perez D, Hauswirth F, Jager D, et al: Protein expression of cancer testis antigens predicts tumor recurrence and treatment response to imatinib in gastrointestinal stromal tumors. Int J Cancer 128: 2947-2952, 2011
7. Svobodova S, Browning J, Macgregor D, et al: Cancer-testis antigen expression in primary cutaneous melanoma has independent prognostic value comparable to that of Breslow thickness, ulceration and mitotic rate. Eur J Cancer 47: 460-469, 2010.

8. Chen CH, Chen GJ, Lee HS, et al: Expressions of cancer-testis antigens in human hepatocellular carcinomas. Cancer Lett 164: 189-195, 2001.

9. Luo G, Huang S, Xie X, et al: Expression of cancer-testis genes in human hepatocellular carcinomas. Cancer Immun 2: 11, 2002.

10. Korangy F, Ormandy LA, Bleck JS, et al: Spontaneous tumor-specific humoral and cellular immune responses to NY-ESO-1 in hepatocellular carcinoma. Clin Cancer Res 10: 4332-4341, 2004.

11. Peng JR, Chen HS, Mou DC, et al: Expression of cancer/testis (CT) antigens in Chinese hepatocellular carcinoma and its correlation with clinical parameters. Cancer Lett 219: 223-232, 2005.

12. Zhang WM, Xiao G, Xie D, Zhang M, Guo AL and Wen JM: [Correlation of NY-ESO-1 gene and protein expression to metastasis and clinicopathologic features of hepatocellular carcinoma]. Ai Zheng 24: 622-626, 2005.

13. Zhang WM, Xiao G, Zhang M, Guo AL, Dong Y and Wen JM: [Expression of NY-ESO-1 and LAGE-1 cancer-testis antigens in hepatocellular carcinoma]. Zhonghua Bing Li Xue Za Zhi 34: 202-205, 2005

14. Nakamura S, Nouso K, Noguchi Y, et al: Expression and immunogenicity of NY-ESO-1 in hepatocellular carcinoma. J Gastroenterol Hepatol 21: 1281-1285, 2006.

15. Lu Y, Wu LQ, Lu ZH, Wang XJ and Yang JY: Expression of SSX-1 and NY-ESO-1 mRNA in tumor tissues and its corresponding peripheral blood expression in patients with hepatocellular carcinoma. Chin Med J (Engl) 120: 1042-1046, 2007.

16. Wang XY, Chen HS, Luo S, Zhang HH, Fei R and Cai J: Comparisons for detecting NY-ESO-1 mRNA expression levels in hepatocellular carcinoma tissues. Oncol Rep 21: 713-719, 2009.

17. Abassi YA, Jackson JA, Zhu J, O'Connell J, Wang X and Xu X: Label-free, real-time monitoring of IgE-mediated mast cell activation on microelectronic cell sensor arrays. J Immunol Methods 292: 195-205, 2004

18. Perez D, Herrmann T, Jungbluth AA, et al: Cancer testis antigen expression in gastrointestinal stromal tumors: new markers for early recurrence. Int J Cancer 123: 1551-1555, 2008.

19. Konishi J, Toyooka S, Aoe M, et al: The relationship between NY-ESO-1 mRNA expression and clinicopathological features in non-small cell lung cancer. Oncol Rep 11: 1063-1067, 2004.

20. Velazquez EF, Jungbluth AA, Yancovitz M, et al: Expression of the cancer/testis antigen NY-ESO-1 in primary and metastatic malignant melanoma (MM)--correlation with prognostic factors. Cancer Immun 7: 11, 2007.

21. Zhou JX, Li Y, Chen SX and Deng AM: Expression and prognostic significance of cancer-testis antigens (CTA) in intrahepatic cholagiocarcinoma. J Exp Clin Cancer Res 30: 2, 2011. 